\title{
Controle da dor no pós-operatório de cirurgia cardíaca: uma breve revisão
}

\section{Control of pain in the postoperative of cardiac surgery: a brief review}

\section{Control del dolor en el postoperatorio de cirugía cardiaca: una breve revisión}

\author{
Luciano Ramos de Lima ${ }^{\mathrm{I}}$, Marina Morato Stival ${ }^{\mathrm{II}}$, Maria Alves Barbosa ${ }^{\mathrm{III}}$, Lílian Varanda Pereira ${ }^{\mathrm{IV}}$
}

\section{RESUMO}

Este estudo teve como objetivo identificar as terapêuticas analgésicas para controle da dor no pós-operatório de pacientes submetidos à cirurgia cardíaca e os instrumentos utilizados para mensurar a experiência dolorosa. Trata-se de uma pesquisa bibliográfica realizada por meio de revisão da literatura nas bases de dados eletrônicos LILACS, revistas eletrônicas da SciELO, dissertações e teses. Foram utilizados os descritores analgesia, analgesia controlada pelo paciente, cirurgia cardíaca, avaliação e dor pós-operatória. O levantamento dos dados ocorreu em maio de 2007 e foram selecionados estudos publicados no período de maio de 1997 a maio de 2007. Na análise realizou-se síntese reflexiva dos estudos. Dos 23 estudos levantados foram utilizados 8 artigos e uma dissertação de mestrado. A técnica de analgesia mais utilizada foi a Analgesia Controlada pelo Paciente (ACP) e os medicamentos mais utilizado foram os opióides, como a morfina, seguidos por antiflamatórios não esteroidais (AINEs) e dipirona. Não foram encontrados estudos que utilizaram a terapêutica não farmacológica. Os instrumentos de avaliação de dor frequentemente utilizados nestes estudos incluíram as escalas unidimensionais como a escala numérica verbal e a escala analógica visual. Ficou também evidenciado que os enfermeiros estão pouco envolvidos em pesquisas que abordam 0 controle da dor no período pós-operatório de cirurgia cardíaca.

Palavras chave: Dor pós-operatória; Cirurgia cardíaca; Medição da dor; Terapêutica; Analgésicos; Avaliação em enfermagem.

\section{ABSTRACT}

This study aimed to identify the analgesic therapeutics which control the pain in the postoperative of cardiac surgery and the instruments to measure this experience. The literature research was conducted by reviewing the literature on databases electronic dada (LILACS), electronic journals (SciELO), dissertations and theses. The following descriptive tools were used: analgesia, patientcontrolled analgesia, cardiac surgery, evaluation and post-surgical pain. The collection data was compiled in May 2007. The selected studies were published during the period from May 1997 to May 2007. In the analysis, it was done a reflexive synthesis of the studies. Among the 23 studies raised, 08 articles and 01 master degree paper were used. The most used analgesia technique was the patient-controlled analgesia (PCA) and the drugs most taken were opiates, such as morphine, anti-inflammatory nonsteroidal drugs (AINEs) and dipyrone. Studies using nonpharmacological therapies were not found. Among the pain evaluation instruments frequently used in these studies were the unidimensional scales such as numerical rating scale and the visual analogue scale. Furthermore, it was clear that the nurses are not completely involved in researches focused on the control of the pain in postoperative period cardiac surgery.

Key words: Postoperative pain; Thoracic surgery; Measurement of pain; Therapeutics; Analgesics; Nursing assessment.

\section{RESUMEN}

Este estudio tuvo como objetivo identificar las terapéuticas analgésicas para control del dolor en el postoperatorio de cirugía cardíaca y los instrumentos utilizados para medida de la intensidad de esa experiencia. Se trata de una investigación exploratoria realizada por medio

\footnotetext{
Bolsista do CNPQ, Enfermeiro mestrando da Universidade Federal de Goiás (UFG), Pós-graduado em UTI pela UFMG. Anápolis/GO. E-mail: enframosll@gmail.com

II Enfermeira, Professora Mestre do Curso de Enfermagem da Faculdade Latino Americana de Anápolis/Go. E-mail: mstival@gmail.com

III Enfermeira, Professora Doutora da Faculdade de Enfermagem UFG, Goiânia/GO. E-mail: maria.malves@gmail.com

IV Enfermeira Orientadora, Professora Doutora da Faculdade de Enfermagem UFG, Goiânia/GO. E-mail: Ivaranda@terra.com.br
} 
Lima LR, Stival MM, Maria Alves Barbosa MA, Pereira LV. Controle da dor no pós-operatório de cirurgia cardíaca: uma breve revisão. Revista Eletrônica de Enfermagem [Internet]. 2008;10(2):521-529. Available from: http://www.fen.ufg.br/revista/v10/n2/v10n2a23.htm

de revisión sistemática de literatura en las bases de datos electrónicos LILACS y en revistas electrónicas de SciELO y disertaciones de maestría and tesis. Fueron utilizados los descriptos analgesia, analgesia controlada por el paciente, cirugía cardíaca, evaluación y dolor postoperatorio. Los datos de la encuesta fue en mayo de 2007, a la que se seleccionaron los estudios publicados en el período comprendido entre mayo de 1997 y mayo de 2007. En el análisis se realizó síntesis reflexiva de los estudios. De los 23 estudios levantados fueron utilizados 8 artículos y una disertación de maestría. La técnica de analgesia más utilizada fue la Analgesia Controlada por el Paciente (ACP) y los medicamentos más utilizados fueron

\section{NTRODUÇÃO}

A dor pós-operatória caracteriza-se como dor aguda prevalente no âmbito hospitalar, comumente associada a dano tecidual. Pode manifestar-se de forma intensa ou moderada em 40 a $60 \%$ dos casos, prevalecendo após cirurgias extensas como as torácicas, abdominais, renais e ortopédicas ${ }^{(1)}$.

Advinda de estimulação nociva intensa ou potencialmente injuriante, a dor aguda geralmente resulta em repercussões funcionais e orgânicas adversas que podem comprometer o resultado do tratamento da lesão original, produzir seqüelas ou mesmo constituir risco de morte. No caso de dor pós-operatória as repercussões incluem complicações neurohumorais, respiratórias e viscerais (gastrointestinais e urológicas), músculoesqueléticas e metabólicas ${ }^{(2)}$.

A ocorrência e a intensidade da dor pósoperatória dependem de fatores constitucionais e relacionados à natureza dos procedimentos operatórios como localização e tipo de incisão, natureza e magnitude do traumatismo e duração das cirurgias. Associam-se, ainda, as complicações cirúrgicas, as características fisiológicas e psicológicas, os aspectos sócioculturais, as experiências prévias com dor, os relacionamentos interpessoais, os fatores ambientais e o preparo/cuidados aos pacientes no período pré-operatório e pós-operatório $(\mathrm{PO})^{(2)}$.

A maioria dos pacientes submetidos a cirurgias de grande porte queixa-se de dor los opiáceos, como la morfina, seguidos por antiinflamatorios no esteroidales (AINEs) y dipirona. No fueron encontrados estudios que utilizaron la técnica analgésica no farmacológica. Los instrumentos de evaluación de dolor sobresaliente en eses estudios incluyeron las escaladas unidimensionales como la escala numérica verbal y la escala analógica visual. Se quedó también evidenciado que los enfermeros están pocos involucrados en investigaciones que aborden el control del dolor en el períodos postoperatorio.

Palabras clave: Dolor postoperatorio; Cirugía torácica; Dimensión del dolor; Terapêutica; Analgésicos; Evaluación en enfermería.

intensa, capaz de gerar complicações pósoperatórias advindas de respiração superficial como a retenção de secreções, atelectasias e processos infecciosos. Além disso, a deambulação tardia e o imobilismo podem resultar em tromboses ${ }^{(3)}$. No caso de cirurgias cardíacas, estudos mostraram que 47 a $75 \%$ dos pacientes relataram algum tipo de dor no $\mathrm{PO}^{(1)}$.

Destaca-se que o manejo da dor no período de recuperação cirúrgica, por meio de terapêuticas farmacológicas e não farmacológicas evita sofrimento desnecessário. Nesse sentido, vale ressaltar que um dos pilares para o adequado alívio da dor inclui a avaliação dessa experiência, uma vez que, se acurada, pode direcionar a escolha de métodos mais eficazes de analgesia, apontar a necessidade de incremento de intervenções ou mesmo de interrupção das já existentes. Contribui de forma importante na elaboração do plano de cuidados e implementação de ações que promovam a identificação precoce da queixa álgica e a pronta intervenção para seu completo alívio $^{(4)}$.

A avaliação da dor no PO de cirurgia cardíaca foi investigada em estudo com 30 pacientes submetidos a cirurgia cardíaca, observando-se intensidade de dor moderada, que permaneceu até o quinto dia $\mathrm{PO}^{(5)}$. Já em outro estudo a dor pós revascularização do miocárdio, por esternotomia, teve intensidade entre 7 (sete) e 10 (dez), mensurada em escala numérica de 0 (zero) a dez (dez), em $49 \%$ dos 
Lima LR, Stival MM, Maria Alves Barbosa MA, Pereira LV. Controle da dor no pós-operatório de cirurgia cardíaca: uma breve revisão. Revista Eletrônica de Enfermagem [Internet]. 2008;10(2):521-529. Available from: http://www.fen.ufg.br/revista/v10/n2/v10n2a23.htm

pacientes em repouso, $78 \%$ deles durante a tosse e em $62 \%$ dos pacientes ao movimento ${ }^{(6)}$.

Nesse contexto, observa-se que apesar dos avanços no manejo da dor serem inquestionáveis, no PO de cirurgia cardíaca essa experiência continua sendo aliviada inadequadamente ${ }^{(7)}$.

Os estudos desenvolvidos nas últimas décadas têm buscado identificar o motivo da persistência dolorosa e do seu inadequado controle visando a solução do problema(7). Outras pesquisas alertaram para o fato da dor pós-operatória ser entendida como uma experiência normal no meio cirúrgico, por faltar aos profissionais conhecimentos básicos sobre a fisiologia da dor e a farmacologia dos analgésicos, além de capacitação para avaliação do quadro álgico, que permanece "invisível", porém deletério ${ }^{(8)}$.

Dentre as terapêuticas para o controle da dor destaca-se a farmacológica. Os antiinflamatórios não esteroidais e os opióides são considerados agentes analgésicos significativos no controle da dor pós-operatória, uma vez que inibem a gênese e a condução do estímulo doloroso, pode-se ser administrados por diferentes vias, por meio de bombas de infusão (BI) e pelo sistema de analgesia controlada pelo paciente $(A C P)$ ou não ${ }^{(9-10)}$.

As terapias não farmacológicas, ou seja, as físicas e cognitivo-comportamentais, incluem um conjunto de medidas de ordem educacional, física, emocional e comportamental, capazes de ativar os centros supra-segmentares responsáveis pela dimensão sensitivodiscriminativa da experiência dolorosa, estimulando, portanto, o sistema supressor de $\operatorname{dor}^{(9)}$.

As terapias físicas incluem a aplicação de calor e frio, massagem, estimulação elétrica e acupuntura e as cognitivo-comportamentais, o relaxamento, distração, imaginação dirigida, hipnose e biofeedback, capazes de promover relaxamento muscular e distração da atenção, o que pode interferir positivamente no controle do estímulo doloroso no pós-operatório ${ }^{(9-10)}$.

A mensuração da dor no meio clínico tem sido feita por meio de instrumentos unidimensionais, destaca-se o uso de escalas ordinais, nas quais são utilizados números (numéricas) ou adjetivos (verbais) como categorias, e as escalas de julgamentos visuais em uma dimensão padrão (VAS - escala analógica visual) ${ }^{(10)}$.

Nas escalas numéricas esse número pode variar de 0 a 100 (até 101 pontos), sendo as de 0 a 5 ( 6 pontos) e de 0 a 10 ( 11 pontos) mais freqüentemente utilizadas. Tais escalas têm a vantagem de serem familiares aos pacientes, uma vez que o ser humano utiliza números desde sua infância. As escalas verbais, nas quais são utilizados adjetivos do tipo leve, moderada, forte, severa ou variantes dessas, são geralmente de 5 ou 6 pontos. Essas também têm a vantagem de serem familiares aos sujeitos visto que utilizam palavras comuns do vocabulário, as quais expressam a dor qualitativamente ${ }^{(10)}$.

As escalas analógicas visuais, mais freqüentemente utilizadas na mensuração da dor pós-operatória, consistem de uma reta de $10 \mathrm{~cm}$ de comprimento, a qual representa o contínuo da experiência dolorosa e possuem em suas extremidades palavras-âncora como: sem dor e pior dor possível. Os sujeitos são instruídos a marcar a intensidade da sensação dolorosa em um ponto dessa linha. Tais escalas têm a vantagem de serem de fácil aplicação, porém, alguns pacientes, especialmente crianças e idosos, encontram certa dificuldade em utilizá-las, o que pode advir da necessidade de certa abstração para compreendê-la. Nestas, os escores de dor podem variar de 0 (zero) a 10 (dez) e são obtidos medindo-se, em milímetros, a distância entre a extremidade ancorada pelas palavras sem dor e a marcação feita pelo paciente ${ }^{(10)}$.

Diante do exposto, julgou-se importante um trabalho de revisão, com o propósito de contribuir com os conhecimentos sobre o controle da dor no pós-operatório de cirurgia cardíaca, com o objetivo de identificar as terapêuticas analgésicas utilizadas no alívio dessa experiência e os instrumentos frequentemente utilizados para mensurá-la.

\section{METODOLOGI A}

Estudo bibliográfico sistematizado, do tipo descritivo e exploratório sobre o controle da dor no pós-operatório de cirurgia cardíaca. 
Lima LR, Stival MM, Maria Alves Barbosa MA, Pereira LV. Controle da dor no pós-operatório de cirurgia cardíaca: uma breve revisão. Revista Eletrônica de Enfermagem [Internet]. 2008;10(2):521-529. Available from: http://www.fen.ufg.br/revista/v10/n2/v10n2a23.htm

A busca bibliográfica incluiu as bases de dados LILACS (Literatura Latino-Americana e do Caribe em Ciências da Saúde) no endereço: http://www.bireme.br/, em revistas eletrônicas disponíveis na base de dados SciELO e em dissertações e teses disponíveis na Biblioteca da Universidade de São Paulo no endereço: http://www.usp.br/.

A revisão bibliográfica foi feita no mês de maio de 2007, revisando-se os estudos desenvolvidos/publicados no período de maio de 1997 a maio de 2007, disponíveis na íntegra nas bases pesquisadas.

A busca seguiu três etapas: na primeira, identificou-se os descritores de assunto: analgesia, analgesia controlada pelo paciente, cirurgia cardíaca, dor pós-operatória, avaliação/medição da dor; na segunda, realizou-se a leitura dos resumos do material encontrado e a seleção daquilo que interessava; na terceira, fez-se uma pré-análise, com leitura dos artigos selecionados, síntese e registro dos dados em um banco de dados construído para esse fim. Os trabalhos foram categorizados pelo assunto, título, ano de publicação, pela base de dados de origem, metodologia, pelos objetivos, resultados e pela conclusão.

\section{RESULTADOS E DISCUSSÃO}

Foram identificados 23 estudos, cuja distribuição por base de dados pode ser visualizada na Tabela 1. Destes, 9 foram selecionados ( 8 artigos científicos e 1 dissertação de mestrado) e analisados para alcance dos objetivos propostos nesse estudo. Nenhuma tese foi encontrada.

Tabela 1: Distribuição dos estudos com foco no controle da dor no pós-operatório de cirurgia cardíaca de acordo com a base de dados consultada. Maio, 2007.

\begin{tabular}{ccccc}
\hline \multirow{2}{*}{ Base de dados } & \multicolumn{2}{c}{ Levantados } & \multicolumn{2}{c}{ Obtidos na íntegra } \\
\cline { 2 - 5 } & $\mathrm{N}$ & $\%$ & $\mathrm{n}$ & $\%$ \\
LILACS & 53 & 100,0 & 8 & 15,1 \\
SciELO & 200 & 100,0 & 13 & 6,5 \\
Biblioteca da USP (dissertações) & 2 & 100,0 & 1 & 100,0 \\
\hline TOTAL & 255 & 100,0 & 23 & 9,0 \\
\hline
\end{tabular}

A distribuição dos 8 artigos e da dissertação de mestrado selecionados, considerando os periódicos em que foram publicados, os autores, o ano de publicação, o tipo de estudo e a associação à Enfermagem pode ser observada no Quadro 1.

Quadro 1: Distribuição dos artigos/dissertação selecionados (9), de acordo com os periódicos, autores, ano de publicação, tipo de estudo e associação à Enfermagem. Maio, 2007

\begin{tabular}{|c|c|c|c|c|}
\hline Periódicos & Autores & Ano & Tipo de estudo & Enfermagem \\
\hline MedUNAB & Rivero; et al. ${ }^{(11)}$. & 2001 & Prospectivo & $\begin{array}{c}\text { Sim } \\
\text { (multiprofissional) }\end{array}$ \\
\hline $\begin{array}{c}\text { Revista Brasileira de } \\
\text { Anestesiologia } \\
\end{array}$ & $\begin{array}{l}\text { Issy; et al. }{ }^{(12)} \text {. Barros; } \\
\text { Lemonica }^{(13)} \text {. }\end{array}$ & $\begin{array}{l}2002 \\
2003 \\
\end{array}$ & $\begin{array}{l}\text { Prospectivo } \\
\text { Retrospectivo } \\
\end{array}$ & $\begin{array}{l}\text { Não } \\
\text { Não } \\
\end{array}$ \\
\hline $\begin{array}{c}\text { Revista Latino-Americana } \\
\text { de Enfermagem }\end{array}$ & Chaves; Pimenta ${ }^{(9)}$. & 2003 & Retrospectivo & Sim \\
\hline $\begin{array}{c}\text { Revista Acta Cirúrgica } \\
\text { Brasileira }\end{array}$ & Xavier; Torres; Rocha ${ }^{(4)}$. & 2005 & $\begin{array}{l}\text { Descritivo, } \\
\text { analítico }\end{array}$ & $\begin{array}{c}\text { Sim } \\
\text { (multiprofissional) }\end{array}$ \\
\hline $\begin{array}{c}\text { Revista do Colégio } \\
\text { Brasileiro de Cirurgiões }\end{array}$ & $\begin{array}{c}\text { Bassanezi; Oliveira } \\
\text { Filho }^{(8)} \text {. }\end{array}$ & 2006 & $\begin{array}{c}\text { Revisão } \\
\text { Bibliográfica }\end{array}$ & Não \\
\hline \multirow[b]{2}{*}{$\begin{array}{l}\text { Brazilian Journal of } \\
\text { Cardiovascular Surgery }\end{array}$} & Borges; et al. ${ }^{(14)}$ & 2006 & $\begin{array}{c}\text { Coorte } \\
\text { experimental }\end{array}$ & Não \\
\hline & $\begin{array}{l}\text { Giacomazzi; Lagni; } \\
\text { Monteiro }^{(5)}\end{array}$ & 2006 & $\begin{array}{c}\text { Coorte, } \\
\text { quantitativo, } \\
\text { longitudinal, } \\
\text { prospectivo }\end{array}$ & Não \\
\hline Dissertação & Autor & Ano & Tipo de estudo & $\begin{array}{l}\text { Associado a } \\
\text { enfermagem }\end{array}$ \\
\hline Mestrado & Bueno $^{(15)}$ & 2006 & $\begin{array}{l}\text { Descritivo, } \\
\text { transversal }\end{array}$ & Sim \\
\hline
\end{tabular}


Lima LR, Stival MM, Maria Alves Barbosa MA, Pereira LV. Controle da dor no pós-operatório de cirurgia cardíaca: uma breve revisão. Revista Eletrônica de Enfermagem [Internet]. 2008;10(2):521-529. Available from: http://www.fen.ufg.br/revista/v10/n2/v10n2a23.htm

Como pode ser observado, quanto ao período de publicação, foram publicados mais estudos no ano de 2006, com 04 estudos, seguido pelo ano de 2003, com 02 estudos. Nos anos 2000, 2002 e 2005 apenas 01 trabalho foi selecionado a cada ano.

Segundo o tipo de estudo identificou-se 01 estudo experimental, 01 estudo de revisão bibliográfica e 07 estudos do tipo descritivo. Todos abordaram o controle da dor pósoperatória por diferentes métodos de analgesia e também a utilização de escalas de mensuração da dor.

Alguns autores afirmaram que, para 0 tratamento da dor é indicada uma abordagem multimodal, que inclui técnicas farmacológicas e não farmacológicas ${ }^{(9,16)}$. Esta abordagem não foi observada nos trabalhos analisados, pois prevaleceram somente as técnicas farmacológicas.

A discussão foi apresentada em três tópicos, incluindo: os estudos que focaram a utilização de terapêuticas analgésicas farmacológicas no pós-operatório de cirurgias cardíacas; os estudos que focaram as terapêuticas analgésicas não farmacológicas no $\mathrm{PO}$ e, os estudos dessa revisão que utilizaram instrumentos unidimensionais e multidimensionais para mensuração da experiência dolorosa no PO de cirurgia cardíaca.

\section{Estudos que utilizaram terapia analgésica farmacológica}

As intervenções farmacológicas são utilizadas como primeira opção no controle da dor aguda, incluindo os antiinflamatórios não esteróidais (AINEs), opióides e ansiolíticos ${ }^{(8,16)}$. Alguns estudos apontam os antidepressivos, anticonvulsivantes e neurolépticos ${ }^{(16)}$. Para dor aguda pós-operatória dos nove estudos analisados observou-se a utilização de opidóides em $55,5 \%$ deles ${ }^{(9,11-13,15)}$.

Em estudo realizado em um hospital privado de São Paulo, do qual participaram 382 pacientes, com idades entre 8 anos e 91 anos, de ambos os sexos, submetidos a cirurgias torácicas, investigou-se o uso de opióides, administrado de diferentes modos ${ }^{(9)}$. Desses pacientes, 332 receberam o analgésico pelo modo intravenoso contínuo (ACP) e 53 pacientes pelo modo endovenoso sem bomba de infusão. Cento e trinta e nove pacientes receberam analgésico pelo modo intravenoso contínuo, associado à dose em bolus; 128 pacientes receberam-no pelo modo intravenoso, com doses em bolus; 41 pacientes receberam analgésicos pelo modo peridural, contínuo, associado às doses em bolus; e 21 receberam pelo modo peridural, por meio de doses em bolus. O método que mais requereu opióides nas 24 horas foi o modo intravenoso contínuo associado à dose em bolus $(18,98 \mathrm{mg})$ e o de menor consumo foi o modo peridural contínuo associado a doses em bolus $(1,15 \mathrm{mg})$. Ainda, $66,2 \%$ dos pacientes receberam uma dose de analgésico complementar. A dor correspondeu a dor leve (1) expressa por meio de escala numérica verbal de 10 pontos. O alívio da dor pós-operatória foi mais adequado para os pacientes que receberam analgésicos pelo modo peridural contínuo associado a doses em bolus e o pior controle foi observado nos pacientes que receberam analgésico pelo modo intravenoso contínuo associado à dose em bolus.

A eficácia da analgesia com opióides, administrados pelo modo ACP, também foi investigado em um estudo envolvendo 368 pacientes submetidos a revascularização do miocárdio, durante 48 horas, com idades entre 10 e 89 anos, de ambos os sexos ${ }^{(11)}$. O analgésico utilizado no $\mathrm{PO}$ em $98,8 \%$ dos casos foi a morfina, com dose média de $60,1 \mathrm{mg}$. Outras drogas foram associadas, como os opióides fracos e os antiiflamatórios não esteróidais (AINEs) em 157 pacientes. A dor foi controlada satisfatoriamente em $97,5 \%$ dos casos, como indicado pelos escores inferiores a 4, atribuídos à intensidade da experiência dolorosa por meio de uma escala analógica visual, de $10 \mathrm{~cm}$ de comprimento, ancorada pelas palavras sem dor e pior dor possível. Ainda, $99,6 \%$ dos pacientes relataram ter sentido dor menor do que o esperado para o processo cirúrgico que haviam sido submetidos.

O efeito do opióide fentanil $50 \mu \mathrm{g} / \mathrm{Kg}$ residual anestésico, por via venosa, foi investigado em 11 pacientes ( 7 homens e 4 mulheres), com média de idade igual a 52 anos, submetidos a revascularização do miocárdio e com níveis plasmáticos de fentanil $>1 \mathrm{ng} / \mathrm{ml}$. A 
Lima LR, Stival MM, Maria Alves Barbosa MA, Pereira LV. Controle da dor no pós-operatório de cirurgia cardíaca: uma breve revisão. Revista Eletrônica de Enfermagem [Internet]. 2008;10(2):521-529. Available from: http://www.fen.ufg.br/revista/v10/n2/v10n2a23.htm

dor foi avaliada durante tosse vigorosa e fisioterapia respiratória complementar, no primeiro e segundo dias de PO, por meio de uma escala numérica de 10 pontos, cujos escores tiveram média de 1,9 a 3,7 no primeiro dia e de 2,1 a 3,8 no segundo dia ${ }^{(12)}$. O fentanil foi complementado por analgésicos como dipirona e tramadol e não foi observada correlação entre intensidade da dor e a concentração plasmática desse opióide, embora os pacientes apresentassem dor leve no estudo, após o segundo dia PO.

A eficácia e segurança do ACP por bomba de infusão, pelo modo peridural, com uso de morfina como terapêutica analgésica, foi comparada a outras vias como a venosa e subcutânea. Participaram do estudo 170 pacientes da cidade de Botucatu, com média de idade de 30 anos, de ambos os sexos, submetidos a cirurgia cardíaca e torácica. A morfina foi o analgésico mais utilizado ( $50 \%)$, seguido pelo tramadol e fentanil. A via peridural foi a de preferência $(49,5 \%)$, com resultados considerados excelentes quanto à qualidade da analgesia, expressa por meio de uma escala numérica verbal, uma escala adjetival e uma escala comportamental. Os resultados mostraram baixos escores atribuídos à intensidade da dor mensurada ${ }^{(13)}$.

No primeiro pós-operatório de cirurgia cardíaca 30 crianças neonatas foram avaliadas quanto ao processo álgico. Oitenta por cento delas recebeu analgesia com citrato de fentanil contínuo, 16,7\% com dipirona internitente e 3,3 $\%$ com citrato de fentanil contínuo e morfina intermitente. A escala utilizada foi de Neonatal Infant Pain Scale (NIPS) que permite identificar e mensurar a dor por meio de observação da expressão facial, comportamental (agitação, choro) e parâmetros fisiológicos (freqüência respiratória e cardíaca, pressão arterial). Os resultados mostraram que entre os indicadores comportamentais, a agitação foi observados em $36 \%$ dos casos, o choro em $20 \%$ deles, e a face contraída em 13,4\%, seguidos pela abertura ocular, movimentação de membros, irritabilidade e parâmetros fisiológicos (taquicardia aparecendo em 13,4\% das vezes e aumento da pressão arterial em $10 \%$ delas, seguidos pela tiragem intercostal, queda de saturação $\mathrm{O}_{2}$ e taquipnéia). $\mathrm{O}$ citrato de fentanil foi 0 analgésico mais utilizado, sendo administrado a $80 \%$ dos neonatos. Quanto à avaliação da dor, $56,7 \%$ dos neonatos foram avaliados sete vezes no pós-operatório, e destes, $56,7 \%$ tiveram ocorrência de dor no primeiro $\mathrm{PO}, 23,3 \%$ tiveram ausência de dor e $20 \%$ não tiveram registros de dor em seu prontuário $^{(15)}$.

A terapêutica medicamentosa é de grande importância para o controle da dor no PO de cirurgia cardíaca. Assim, pesquisadores também desenvolveram estudos com o objetivo de investigar a analgesia obtida por meio de drogas para o controle dessa experiência como os AINEs (dipirona, acetaminofeno) e opióides (tramadol, morfina, fentanil, nalbufina entre outros) destaca-se os últimos como os mais indicados para controle da dor pós-operatória ${ }^{(8)}$. Ainda, outros pesquisadores também utilizaram morfina em $98 \%$ dos pacientes, seguida de tramadol e dipirona com controle da dor satisfatório em $97,5 \%$ dos pacientes nas primeiras 48 horas $^{(11)}$.

Alguns dos estudos apresentados no Quadro 1 não relataram o tipo de analgesia medicamentosa utilizada durante a avaliação $^{(4-}$ $5,14)$.

O controle medicamentoso da dor pósoperatória pode ser realizado por diferentes vias e modos de administração dos analgésicos. As principais vias incluem a sublingual, oral, transdérmica, parenteral (subcutâneo, intramuscular e intravenoso) e epidural, por meio de sistemas semi ou totalmente implantáveis ${ }^{(15)}$.

Entre os estudos analisados destaca-se a utilização freqüente da técnica de ACP por via intravenosa ${ }^{(9,8,11,13,15)}$. Os resultados concordam com pesquisa similar que destacou ser esse o método preferencialmente indicado para controle da dor aguda pós-operatória, especialmente se associado ao uso de uma BI eletrônica ${ }^{(16)}$. Outros pesquisadores também defendem o uso de ACP, por qualquer via, com utilização de opióides, pois se considerou que poderia evitar flutuações nas concentrações plasmáticas do analgésico utilizado, resultando em analgesia mais eficiente ${ }^{(8)}$. Outro aspecto referido nos estudos é que a 
Lima LR, Stival MM, Maria Alves Barbosa MA, Pereira LV. Controle da dor no pós-operatório de cirurgia cardíaca: uma breve revisão. Revista Eletrônica de Enfermagem [Internet]. 2008;10(2):521-529. Available from: http://www.fen.ufg.br/revista/v10/n2/v10n2a23.htm

utilização/associação de mais de uma droga como analgésico, empregadas em doses menores, o que constitui a analgesia multimodal ou balanceada. Também foram consideradas efetivas as técnicas analgésicas como ACP, analgesia epidural e técnicas de analgesia regional. Em outro estudo foi evidenciada a associação entre ACP e bem estar PO de pacientes submetidos a cirurgia cardíaca, defendendo-se o uso de cateteres peridurais e a técnica ACP, com morfina, como as mais seguras e eficazes ${ }^{(11)}$.

\section{Estudos que utilizaram a terapêutica analgésica não farmacológica \\ A analgesia não} farmacológica/medicamentosa inclui os métodos físicos - aplicação de calor e de frio superficial, técnicas de estimulação elétrica aplicadas à pele, massagens, uso de repouso, exercícios de alongamento e as cognitivocomportamentais ${ }^{(16)}$.

Tais terapêuticas não foram utilizadas nos estudos analisados nesta revisão. Os autores inferem que pode haver certa resistência dos profissionais em utilizar tais técnicas por tratarse de pacientes submetidos a cirurgias cardíacas, classificadas como de grande porte, que exigem a utilização de aparelhos mais sofisticados, sensores e drenos, que conectados ao corpo dos pacientes, poderiam interferir em sua mobilidade.

Alguns autores afirmaram que para o tratamento da dor é indicada uma abordagem analgésica multimodal, que inclua terapêuticas farmacológicas e não farmacológicas ${ }^{(9,16)}$. Essa modalidade não foi evidenciada nos trabalhos identificados, pois foram apontadas somente as técnicas farmacológicas.

\section{Estudos que utilizaram escalas de mensuração da dor}

A mensuração da dor, na maioria dos estudos (90\%) desta revisão (Quadro 2 ), foi realizada por meio de instrumentos unidimensionais, utilizados para mensuração de apenas uma dimensão dessa experiência ${ }^{(5,11-16)}$. Tais escalas são frequentemente utilizadas para medir a intensidade da dor, e incluem as escalas numéricas (de 0 a 10), escala de descritores verbais (sem dor, dor leve, dor moderada, dor intensa, dor insuportável), escala analógica visual, escala de copos e escala de faces de adultos ${ }^{(16-17)}$.

Quadro 2: Distribuição dos estudos segundo o tipo de escala utilizada para mensuração da dor (unidimensional e multidimensional) e resultado encontrado. Maio, 2007.

\begin{tabular}{|c|c|c|}
\hline $\begin{array}{c}\text { Uso de única escala } \\
\text { unidimensional }\end{array}$ & Estudo & Resultado encontrado \\
\hline \multirow{2}{*}{ Escala numérica verbal (ENV) } & Chaves; Pimenta $^{(9)}$ & Dor leve (1) \\
\hline & Issy et al. ${ }^{(12)}$ & Menor de $(4,5)$ \\
\hline \multirow{2}{*}{ Escala visual analógica (EVA) } & Rivero et al. ${ }^{(11)}$ & Inferior a (4) \\
\hline & Borges et al. ${ }^{(14)}$ & Dor moderada, mediana (3) \\
\hline $\begin{array}{c}\text { Uso de mais de uma escala } \\
\text { unidimensional }\end{array}$ & Estudo & Resultado encontrado \\
\hline Desenho corpo humano & \multirow{2}{*}{ Giacomazzi; Lagni; Monteiro ${ }^{(5)}$} & Externo \\
\hline Escala subjetiva analógica & & Dor moderada \\
\hline ENV & \multirow{3}{*}{ Barros; Lemomica ${ }^{(13)}$} & Níveis baixos de dor média 0,99 \\
\hline Adjetival & & - \\
\hline Comportamental & & - \\
\hline Comportamentais & \multirow{3}{*}{ Bueno $^{(15)}$} & Agitação 36\%, choro $20 \%$ \\
\hline Fisiológicos & & $\begin{array}{c}\text { Taquicardia } 13,4 \% \text {; aumento } \\
\text { pressão arterial } 10 \%\end{array}$ \\
\hline Escala NIPS & & $56,7 \%$ utilizaram a escala \\
\hline $\begin{array}{c}\text { Uso de mais de uma escala } \\
\text { associação entre escalas } \\
\text { unidimensional e } \\
\text { multidimensional }\end{array}$ & Estudo & Resultado encontrado \\
\hline ENV & \multirow{2}{*}{ Xavier; Torres; Rocha ${ }^{(4)}$} & Média 5,7 \\
\hline Questionário de McGill & & 11 a 20 pontos \\
\hline
\end{tabular}


Lima LR, Stival MM, Maria Alves Barbosa MA, Pereira LV. Controle da dor no pós-operatório de cirurgia cardíaca: uma breve revisão. Revista Eletrônica de Enfermagem [Internet]. 2008;10(2):521-529. Available from: http://www.fen.ufg.br/revista/v10/n2/v10n2a23.htm

Em 44,4\% dos estudos dessa revisão observou-se a utilização de uma única escala para mensuração da intensidade da dor. As mais utilizadas foram as escalas unidimensionais - escala numérica verbal (ENV) e a escala visual analógica (EVA) ${ }^{(9,11-12,14)}$. Pesquisadores afirmaram que são as escalas mais utilizadas no período $\mathrm{PO}$, porém, como instrumentos unidimensionais quantificam a dor em apenas uma dimensão, porém, são criticados por não possibilitarem mensuração multidimensional dessa experiência ${ }^{(18)}$. São de fácil aplicabilidade e requerem pouco tempo do observador e do paciente para a tarefa de mensuração, bem como, baixos custos ${ }^{(19)}$.

Escalas que utilizam descritores verbais são consideradas vantajosas quando permitem mensurar diferentes dimensões da experiência dolorosa, sendo utilizadas na mensuração da dor clínica e experimental, refletindo experiências subjetivas ${ }^{(20)}$.

A utilização de mais de uma escala unidimensional para avaliação da dor foi observada em $33,3 \%$ dos estudos, incluindo os diagramas corporais; escala subjetiva analógica visual, escala numérica, escala adjetival, escala comportamental e de indicadores fisiológicos (NIPS) e $11,1 \%$ dos estudos associou escalas unidimensionais (ENV) com multidimensionais (Questionário de McGill) ${ }^{(4,5,13,15)}$. Assim, a utilização de mais de uma escala vem de encontro à opinião de alguns pesquisadores, que defendem sua importância na validação das escalas e evidencia da fidedignidade dos dados obtidos $^{(4,5,13,15)}$.

Alguns instrumentos multidimensionais permitem investigar a descrição da dor por meio de palavras denominadas descritores de dor. Trazem dados tanto qualitativos como quantitativos $^{(17)}$. O questionário McGill corresponde a um desses instrumentos sendo utilizado para avaliação dos aspectos sensitivos, afetivos e avaliativos da dor. Inclui um diagrama corporal para localização da experiência dolorosa, uma escala de intensidade de descritores verbais e 78 descritores de dor agrupados em 4 grandes grupos e 20 subgrupos $^{(19)}$.

\section{CONCLUSÃO}

O controle da dor pós-operatória é um aspecto importante do cuidado no meio cirúrgico, com vistas a diminuir e controlar as complicações pós-operatórias e garantir uma recuperação mais rápida e sem sofrimento.

Nessa revisão, apontou-se a utilização de terapêuticas medicamentosas para o controle da dor. A ACP foi o modo mais frequentemente utilizado para administração dos analgésicos, despontando como método seguro e eficaz na obtenção e manutenção da analgesia.

As terapêuticas não farmacológicas não foram identificadas entre os estudos analisados. Acredita-se que tal fato deveu-se principalmente à insegurança dos profissionais em utilizar tais terapêuticas no período pósoperatório de cirurgia cardíaca, pelo temor em prejudicar os pacientes, já que muitos estão em estado grave e com a mobilidade física prejudicada.

As drogas mais utilizadas foram os opióides (morfina, tramadol, fentanil), seguidos por AINEs e dipirona, muitas vezes associados a outras drogas, visando a melhoria do controle da dor.

Em relação aos instrumentos de medida, prevaleceram as escalas unidimensionais como a ENV e a EVA. Apenas em um estudo observou-se a utilização de instrumentos multidimensionais como o Questionário de McGill.

Aponta-se finalmente, 0 pequeno envolvimento de enfermeiros em pesquisas que abordam o controle da dor no pós-operatório de cirurgia cardíaca, fato observado também em outros tipos de estudos sobre o controle dessa experiência.

\section{REFERÊNCI AS}

1. Chung JWY, Lui JCZ. Postoperative pain management: study of patients level of pain and satisfaction with health are providers responsiveness to their reports of pain. Nurs Health Sciences. 2003;3(10):295-299.

2. Teixeira MJ, Valverde Filho J. Dor aguda. In: Teixeira MJ. Dor contexto interdisciplinar. $1^{a}$ edição. Curitiba: Editora Maio; 2003.

3. Boisseau N, Rabary O, Padovani B, Staccini P, Mouroux J. Improvement of dynamic analgesia 
Lima LR, Stival MM, Maria Alves Barbosa MA, Pereira LV. Controle da dor no pós-operatório de cirurgia cardíaca: uma breve revisão. Revista Eletrônica de Enfermagem [Internet]. 2008;10(2):521-529. Available from: http://www.fen.ufg.br/revista/v10/n2/v10n2a23.htm

does not decrease atelectasias after thoracotomy. Br J Anaesth. 2001;87(1): 564-9. 4. Xavier TT, Torres, GV, Rocha VM. Dor pósoperatória: características quanti-qualitativa relacionadas a toracotomia póstero-lateral e esternotomia. Acta Cirúrgica Brasileira [Internet]. 2005 [cited 2007 mar 13];20 Supl.1:63-8. Available from: http://www.scielo.br/pdf/acb/v20s1/25570.pdf

5. Giacomazzi CM, Lagni VB, Monteiro MB. A dor pós-operatória como contribuinte do prejuízo na função pulmonar em pacientes submetidos à cirurgia cardíaca Braz. J Cardiovasc. Surg [Internet]. 2006 [cited 2007 set 11];21(4):38692. Available

from: http://www.scielo.br/pdf/bjcvs/v21n4/a08v21n4.pdf 6. Lahtinen $\mathrm{P}$, Kokki, HMD, Hynynen M. Pain after cardiac surgery: a prospective Cohort study of 1-year. Incidence and intensity. Anesthesiology. 2006; 105(3):794-800.

7. Silva MAS. Efeitos da intervenção treinamento, avaliação e registro sistematizado no controle da dor no pós-operatório de cirurgia cardíaca [dissertação]. [São Paulo]: Escola de Enfermagem da Universidade de São Paulo, 2007. 129 p.

8. Bassanezi BSB, Oliveira Filho AG. Analgesia pós-operatória. Rev Col Bras Cir [Internet]. 2006 [cited 2007 jan 20];33(2):116-22. Available from: http://www.scielo.br/pdf/rcbc/v33n2/v33n2a11.pdf 9. Chaves LD, Pimenta CAM. Controle da dor pós-operatória: comparação entre métodos analgésicos. Rev Latino-am Enfermagem [Internet]. 2003 [cited 2007 jan 14];11(2):2159. Available

from:

http://www.scielo.br/pdf/rlae/v11n2/v11n2a11.pdf

10. Melzack R, Katz J. Pain measurement in persons. In: Wall PD, Melzack R. Editores. Textbook of pain. $3^{a}$ edição. Edinburgh: Churchill Livingstone; 1994. p. 337-56.

11. Rivero OFG, Suárez MPP, Martínez RG, Palomino CV, Sossa ÓA, Bottía JM et al. Analgesia controlada por el paciente (PCA) en cirugía carditorácica. Experienci de 13 anos de la Fundación Cardivascular del Oriente Colombiano. MEDUNAB. [Internet] 2001 [cited 2007 jun 15]; 4(10):1-7 Available from: http://caribdis.unab.edu.co/pls/portal/docs/PAG E/REVISTAMEDUNAB/NUMEROSANTERIORES/R EVISTA410/PCA.PDF
12. Issy AM, Espada EB, Sakata RK, Lanchote VL, Auler Júnior JOC, Santo SRCJ. Efeito Analgésico Residual do Fentanil em Pacientes Submetidos a Revascularização do Miocárdio com Circulação Extracorpórea. Rev Bras Anestesiol [Internet]. 2002 [cited 2007 out 22];52(52):562-9. Available from: http://www.scielo.br/pdf/rba/v52n5/v52n5a06.pdf

13. Barros GAM, Lemonica L. Considerações sobre analgesia controlada pelo paciente em hospital universitário. Rev Bras Anestesiol [Internet] 2003 [cited $2007 \mathrm{fev} 20$ ]; 53(1):6982. Available from: http://www.scielo.br/pdf/rba/v53n1/v53n1a10.pdf 14. Borges JBC, Ferreira DLMP, Carvalho SMR, Martins AS, Andrade RR, Silva MAM. Avaliação da intensidade de dor e da funcionalidade no pós-operatório recente de cirurgia cardíaca Braz J Cardiovasc Surg [Internet]. 2006 [cited 2007 mar 15];21(4):393-402. Available from: http://www.scielo.br/pdf/bjcvs/v21n4/a09v21n4.pdf 15. Bueno $M$. Dor e analgesia em recémnascidos submetidos a cirurgia cardíaca. [dissertação]. [São Paulo]: Escola de Enfermagem/USP; 2006. 151 p.

16. Chaves LD. Considerações de enfermagem na analgesia controlada pelo paciente. Prática Hospitalar. 2005;(39):29-32.

17. Pereira LV, Sousa, FAEF. Mensuração e avaliação da dor pós-operatória: uma breve revisão. Rev Latino-am Enfermagem. 1998;6(3):77-84.

18. Calil AM, Pimenta CAM. Intensidade da dor e adequação de analgesia. Rev Latino-am Enfermagem. 2005;13(5):692-9.

19. Andrade FA, Pereira LV, Sousa FAEF. Mensuração da dor no idoso: uma revisão. Rev Latino-am Enfermagem. 2006;14(2):271-6.

20. Pereira LV, Sousa FAEF. Psychophysical evaluation of the descriptors of pain in the postoperative. Rev Latino-am Enfermagem. 2007;15(3):474-9.

Artigo recebido em 23.08.07

Aprovado para publicação em 30.06.08 